\title{
Intuitionistic fuzzy index matrix representation of color images
}

\author{
R.K. Nivendhaa ${ }^{1}$ and R. Parvathi ${ }^{2}$ \\ ${ }^{1}$ Department of Mathematics, Vellalar College for Women \\ Erode, Tamilnadu, India \\ e-mail: nivedhaakaran@gmail.com \\ ${ }^{2}$ Department of Mathematics, Vellalar College for Women \\ Erode, Tamilnadu, India \\ e-mail: paarvathis@rediffmail.com
}

Received: 1 September 2020 Revised: 8 November 2020 Accepted: 15 November 2020

\begin{abstract}
It is usual in image processing that binary (black \& white) and gray images are represented by crisp sets and fuzzy sets respectively. In this paper, an attempt has been made to represent a color image (RGB) using intuitionistic fuzzy index matrices. The objective of this representation is to apply mathematical operators on intuitionistic fuzzy index matrices in processing RGB images.
\end{abstract}

Keywords: 3D - index matrix, 3D - intuitionistic fuzzy index matrices, 3 folded - intuitionistic fuzzy index matrix, Image representation.

2010 Mathematics Subject Classification: 03E72, 11C20, 68U10.

\section{Introduction}

In computer science, the representation of an image can take many forms. Most of the time, it refers to the way that the carried information, such as color, is coded digitally and how the image is stored. Each pixel has a specific color or shade of gray, and in combination with neighboring pixels it creates the illusion of a constant tone image. Image processing, as its name suggests, is the processing of an image to improve its quality.

Binary image is an image that consists of only black and white pixels. Technically, these types of images are called as black (0) and white (1) Image. Grayscale image refers to as 
black-and-white (as in old photos) are actually grayscale. It contains intensity values ranging from a minimum (depicting absolute black) to a maximum (depicting absolute white) and in between varying shades of gray, ranges between 0 and 255 .

Color image: It is known that such an image is composed of the three primary colors, Red, Green and Blue, hence also called an RGB image. RGB value: All colors in real life can be made by combining red, blue and green components in varying proportions. This triplet has each value ranging from 0 to 255 , with 0 obviously meaning no component of that particular color and 255 meaning full component. In MATLAB, a RGB image is basically an $M \times N \times 3$ array of color pixel, where each color pixel is associated with three values which corresponds to red, blue and green color component of RGB image at a specified spatial location.

Image represented in the RGB color model consist of three component images. When fed into an RGB monitor, these three images combine on the screen to produce a composite color image. The number of bits used to represent each pixel in RGB space is called the pixel depth. Consider an RGB image in which each of the red, green and blue images are an 8- bit image. Under these conditions each RGB color pixel that is, a triplet of values $(R, G, B)$ is said to have a depth of 24 bits. In [3], the concept of 2D-Index Matrix (IM) is introduced and discussed in detail. In [5], the basic definition of 3D-IM is given. Three Dimensional Intuitionistic Fuzzy Index Matrices (IFIMs) are introduced in [5]. In the literature review, it is observed by the authors that any form of IF concepts are not yet used to represent an RGB image. The authors got motivated to introduce 3-folded intuitionistic fuzzy index matrixes for representing RGB images. In Section 2, the definition of 3D-IMs and 3D-IFIMs are given. Section 3 deals with 3-folded intuitionistic fuzzy index matrix representation (IFIMR) of RGB images with six combinations and illustrated through a numerical example.

\section{Basic definitions}

In this section, basic definitions of three dimensional index matrix [2] and three dimensional intuitionistic fuzzy index matrixes [5] which are necessary for this work, are presented.

Definition 2.1 [2]. Let $I$ be a fixed set of indices and $\chi$ be a fixed set of objects. The three dimensional index matrix (3D-IM) with index sets $K, L$ and $H(K, L, H \subset I)$, is an object of the form:

$$
\begin{aligned}
& {\left[K, L, H,\left\{a_{k_{i}, l_{j}, h_{g}}\right\}\right] \equiv}
\end{aligned}
$$

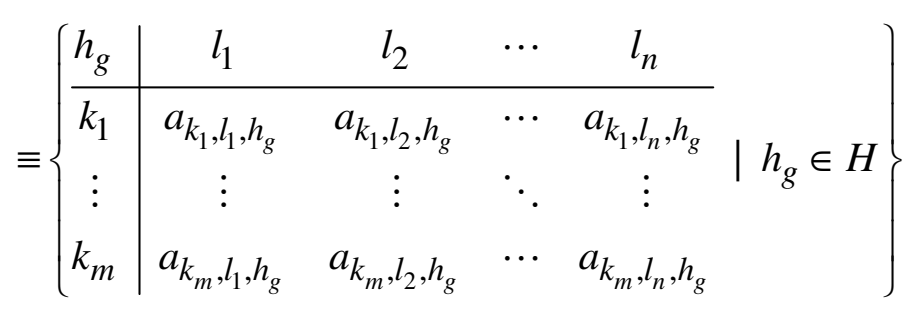




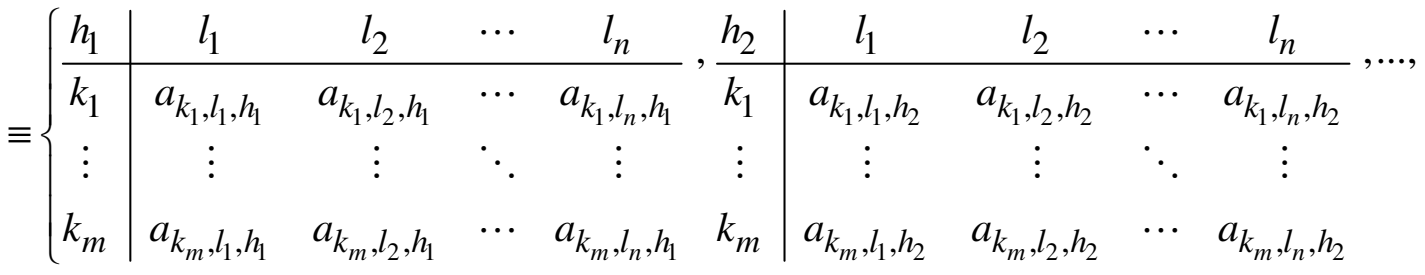

$$
\begin{aligned}
& \left.\begin{array}{c|cccc}
h_{f} & l_{1} & l_{2} & \cdots & l_{n} \\
\hline k_{1} & a_{k_{1}, l_{1}, h_{f}} & a_{k_{1}, l_{2}, h_{f}} & \cdots & a_{k_{1}, l_{n}, h_{f}} \\
\vdots & \vdots & \vdots & \ddots & \vdots \\
k_{m} & a_{k_{m}, l_{1}, h_{f}} & a_{k_{m}, l_{2}, h_{f}} & \cdots & a_{k_{m}, l_{n}, h_{f}}
\end{array}\right\} \text {, }
\end{aligned}
$$

where $K=\left\{k_{1}, k_{2}, k_{3}, \ldots, k_{m}\right\}, L=\left\{l_{1}, l_{2}, l_{3}, \ldots, l_{n}\right\}, H=\left\{h_{1}, h_{2}, h_{3}, \ldots, h_{f}\right\}$, and for $1 \leq i \leq m$, $1 \leq j \leq n, 1 \leq g \leq f: a_{k_{i}, l_{j}, h_{g}} \in \chi$.

Definition 2.2 [1]. Let $I$ be a fixed set. By 3D-IFIM with index sets $K, L$ and $H(K, L, H \subset I)$, we denote the object:

$$
\left[K, L, H,\left\{\left\langle\mu_{k_{i}, l_{j}, h_{g}}, v_{k_{i}, l_{j}, h_{g}}\right\rangle\right\}\right] \equiv
$$

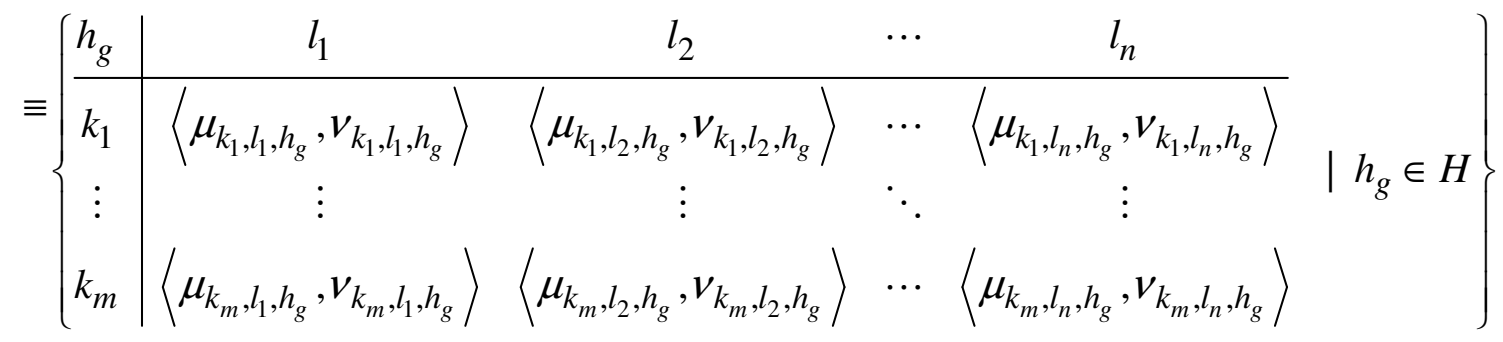

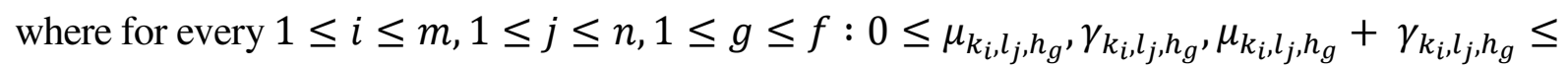
$1, K=\left\{k_{1}, k_{2}, k_{3}, \ldots k_{m}\right\}, L=\left\{l_{1}, l_{2}, l_{3}, \ldots . l_{n}\right\}, H=\left\{h_{1}, h_{2}, h_{3}, \ldots \ldots h_{f}\right\}$.

\section{Main results}

For representing 2D color images, the elements of the matrix (2.1) need different notations as given in Definition 3.1.

Definition 3.1. Let $I$ be a fixed set. By 3D-IFIMR with index sets $K, L$ and $H(K, L, H \subset I)$, we denote the object:

$$
\left[K, L, H,\left\{\left\langle\mu_{k_{i j}, l_{i j}, h_{i j}}, v_{k_{i j}, l_{i j}, h_{i j}}\right\rangle\right\}\right] \equiv
$$




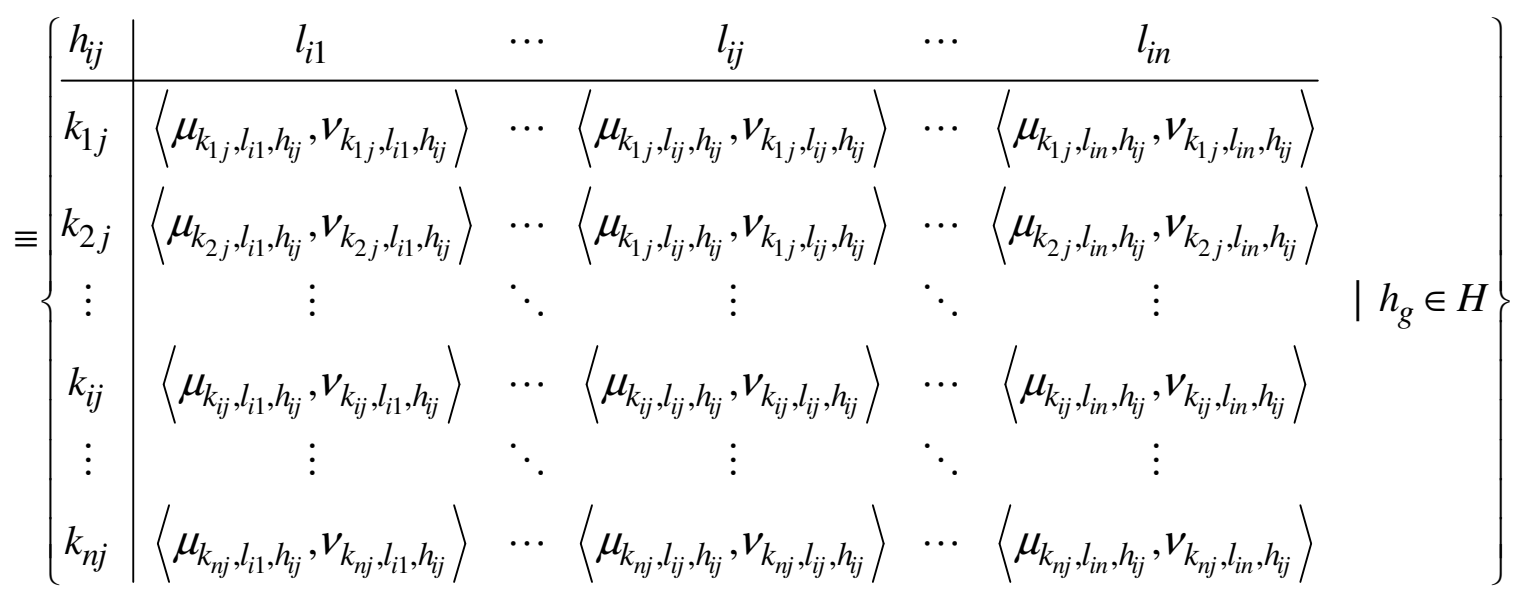

where for every $1 \leq i \leq n, 1 \leq j \leq n$, it holds that $0 \leq \mu_{k_{i j}, l_{i j}, h_{i j}}, v_{k_{i j}, l_{i j}, h_{i j}}, \mu_{k_{i j}, l_{i j}, h_{i j}}+v_{k_{i j}, l_{i j}, h_{i j}} \leq 1$, $K=\left\{k_{i j}\right\}, L=\left\{l_{i j}\right\}, H=\left\{h_{i j}\right\}$.

For the sake of convenience, the definition of 3D-IFIMR is used in a different way to derive a 3-folded-intuitionistic fuzzy index matrix representation (3f-IFIMR) of RGB images, as follows.

Definition 3.2. Let $I$ be a fixed set. By 3f-IFIMR of rectangular RGB images with index sets $R, G$ and $B(R, G, B \subset\{0,1,2, \ldots, 255\})$, we denote the object:

$$
\left.\equiv \begin{array}{c|cccc}
b_{i j} & g_{i 1} & g_{i 2} & \cdots & g_{i n} \\
\hline r_{1 j} & \left\langle\mu_{r_{11}}, v_{g_{11}}, \pi_{b_{11}}\right\rangle & \left\langle\mu_{r_{12}}, v_{g_{12}}, \pi_{b_{12}}\right\rangle & \ldots & \left\langle\mu_{r_{1 n}}, v_{g_{1 n}}, \pi_{b_{1 n}}\right\rangle \\
r_{2 j} & \left\langle\mu_{r_{21}}, v_{g_{21}}, \pi_{b_{21}}\right\rangle & \left\langle\mu_{r_{22}}, v_{g_{22}}, \pi_{b_{22}}\right\rangle & \ldots & \left\langle\mu_{r_{2 n}}, v_{g_{2 n}}, \pi_{b_{2 n}}\right\rangle \\
\vdots & \vdots & \vdots & \ddots & \vdots \\
r_{m j} & \left\langle\mu_{r_{m 1}}, v_{g_{m 1}}, \pi_{b_{m 1}}\right\rangle & \left\langle\mu_{r_{m 2}}, v_{g_{m 2}}, \pi_{b_{m 2}}\right\rangle & \ldots & \left\langle\mu_{r_{m n}}, v_{g_{m n}}, \pi_{b_{m n}}\right\rangle
\end{array}\right\}
$$

where for every $1 \leq i \leq m, 1 \leq j \leq n$, it holds that $0 \leq \mu_{r_{i j}}, v_{g_{i j}}, \pi_{b_{i j}} \leq 1,0 \leq \mu_{r_{i j}}+v_{g_{i j}}+\pi_{b_{i j}} \leq 3$, and $R=\left\{r_{i j}\right\}, G=\left\{g_{i j}\right\}, B=\left\{b_{i j}\right\}$ for every $i$ and $j$ are crisp subsets of $\{0,1,2, \ldots, 255\}$.

\section{Note:}

i. Among $R, G, B$ colors, in each pixel, when one color is considered as member, the other two colors are to be considered as non-member and hesitant. Thus, the following six ways are used to represent an RGB image:
1) $\left[R, G, B,\left\{\left\langle\mu_{r_{i j}}, v_{g_{i j}}, \pi_{b_{i j}}\right\rangle\right\}\right.$,
2) $\left[R, B, G,\left\{\left\langle\mu_{r_{i j}}, v_{b_{i j}}, \pi_{g_{i j}}\right\rangle\right\}\right.$,
3) $\left[G, R, B,\left\{\left\langle\mu_{g_{i j}}, v_{r_{i j}}, \pi_{b_{i j}}\right\rangle\right\}\right.$,
4) $\left[G, B, R,\left\{\left\langle\mu_{g_{i j}}, v_{b_{i j}}, \pi_{r_{i j}}\right\rangle\right\}\right.$,
5) $\left[B, G, R,\left\{\left\langle\mu_{b_{i j}}, v_{g_{i j}}, \pi_{r_{i j}}\right\rangle\right\}\right.$,
6) $\left[B, R, G,\left\{\left\langle\mu_{b_{i j}}, v_{r_{i j}}, \pi_{g_{i j}}\right\rangle\right\}\right.$.

ii. According to the ways of representation the notations may be changed. 


\subsection{Numerical example}

This section is devoted to understanding the $3 \mathrm{f}$-IFIMR concept with the help of a numerical example. To illustrate, an RGB image in Fig. 1 is considered. The image is read using MATLAB and the matrices corresponding to Red, Green, and Blue colors are displayed in matrices (3.3), (3.4), and (3.5) respectively.

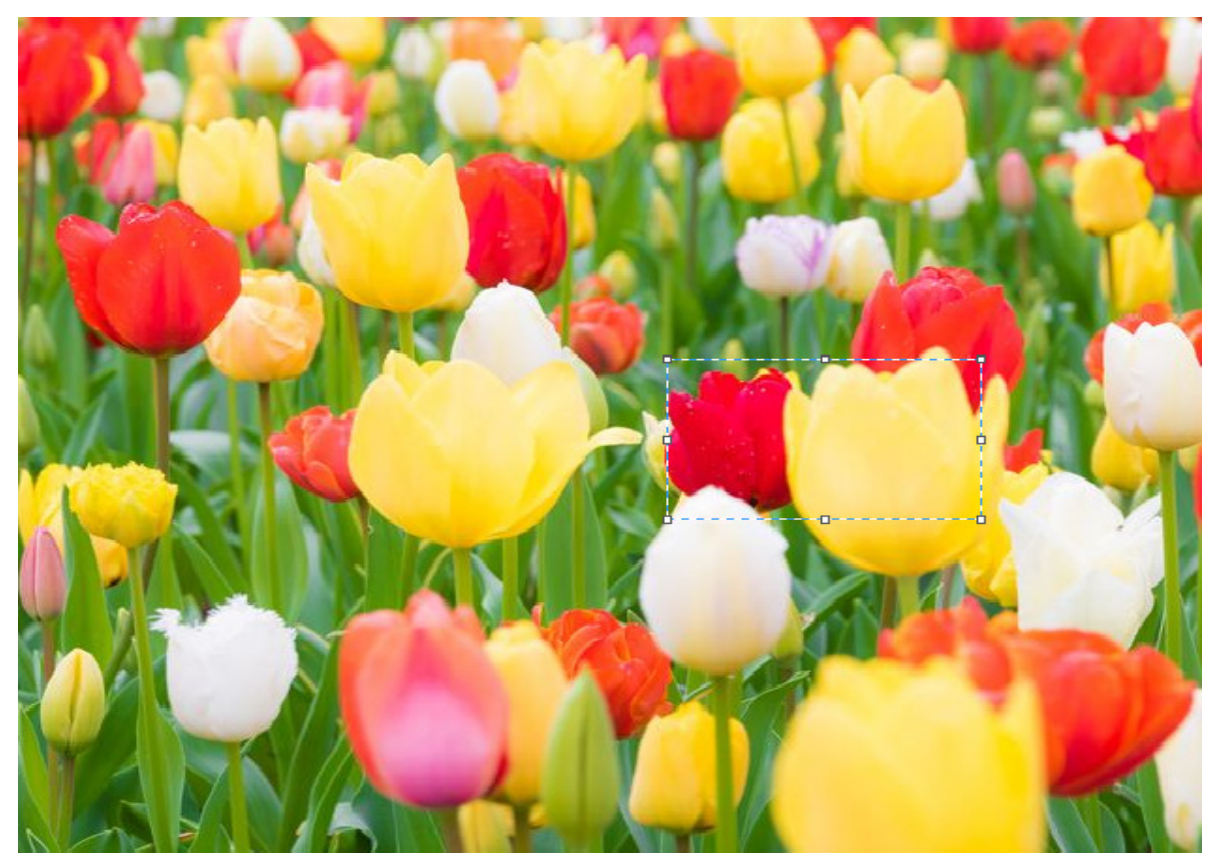

Figure 1. RGB image

$$
\begin{gathered}
R=\left[\begin{array}{lll}
r_{11} & r_{12} & r_{13} \\
r_{21} & r_{22} & r_{23} \\
r_{31} & r_{32} & r_{33} \\
r_{41} & r_{42} & r_{43}
\end{array}\right]=\left[\begin{array}{lll}
173 & 211 & 247 \\
241 & 255 & 252 \\
218 & 251 & 253 \\
205 & 232 & 254
\end{array}\right] \\
G=\left[\begin{array}{lll}
g_{11} & g_{12} & g_{13} \\
g_{21} & g_{22} & g_{23} \\
g_{31} & g_{32} & g_{33} \\
g_{41} & g_{42} & g_{43}
\end{array}\right]=\left[\begin{array}{ccc}
91 & 174 & 181 \\
24 & 191 & 226 \\
15 & 184 & 232 \\
149 & 192 & 230
\end{array}\right] \\
B=\left[\begin{array}{lll}
b_{11} & b_{12} & b_{13} \\
b_{21} & b_{22} & b_{23} \\
b_{31} & b_{32} & b_{33} \\
b_{41} & b_{42} & b_{43}
\end{array}\right]=\left[\begin{array}{ccc}
82 & 119 & 126 \\
66 & 114 & 127 \\
42 & 106 & 118 \\
143 & 122 & 121
\end{array}\right]
\end{gathered}
$$

From Definition 3.1, the RGB image in Fig. 1 is represented as given in (3.6). 


$$
\begin{aligned}
& {\left[R, G, B,\left\{\mu_{r_{i j}}, v_{g_{i j}}, \pi_{b_{i j}}\right\}\right] \equiv} \\
& \equiv \begin{array}{c|ccc}
b_{i j} & g_{i 1} & g_{i 2} & g_{i 3} \\
\hline r_{1 j} & \left\langle\mu_{r_{11}}, v_{g_{11}}, \pi_{b_{11}}\right\rangle & \left\langle\mu_{r_{12}}, v_{g_{12}}, \pi_{b_{12}}\right\rangle & \left\langle\mu_{r_{13}}, v_{g_{13}}, \pi_{b_{13}}\right\rangle
\end{array} \\
& r_{2 j}\left\langle\left\langle\mu_{r_{21}}, v_{g_{21}}, \pi_{b_{21}}\right\rangle\left\langle\mu_{r_{22}}, v_{g_{22}}, \pi_{b_{22}}\right\rangle\left\langle\mu_{r_{23}}, v_{g_{23}}, \pi_{b_{23}}\right\rangle\right. \\
& r_{3 j}\left\langle\left\langle\mu_{r_{31}}, v_{g_{31}}, \pi_{b_{31}}\right\rangle\left\langle\mu_{r_{32}}, v_{g_{32}}, \pi_{b_{32}}\right\rangle\left\langle\mu_{r_{33}}, v_{g_{33}}, \pi_{b_{33}}\right\rangle\right. \\
& r_{4 j} \mid\left\langle\mu_{r_{41}}, v_{g_{41}}, \pi_{b_{41}}\right\rangle\left\langle\mu_{r_{42}}, v_{g_{42}}, \pi_{b_{42}}\right\rangle\left\langle\mu_{r_{43}}, v_{g_{43}}, \pi_{b_{43}}\right\rangle \\
& \equiv \begin{array}{c|ccc}
b_{i j} & g_{i 1} & g_{i 2} & g_{i 3} \\
\hline r_{1 j} & \left\langle\mu_{173}, v_{91}, \pi_{82}\right\rangle & \left\langle\mu_{211}, v_{174}, \pi_{119}\right\rangle & \left\langle\mu_{247}, v_{181}, \pi_{126}\right\rangle
\end{array} \\
& r_{2 j}\left\langle\left\langle\mu_{241}, v_{24}, \pi_{66}\right\rangle\left\langle\mu_{255}, v_{191}, \pi_{114}\right\rangle\left\langle\mu_{252}, v_{226}, \pi_{127}\right\rangle\right. \\
& r_{3 j}\left\langle\mu_{218}, v_{15}, \pi_{42}\right\rangle\left\langle\mu_{251}, v_{184}, \pi_{106}\right\rangle\left\langle\mu_{253}, v_{232}, \pi_{118}\right\rangle \\
& r_{4 j} \mid\left\langle\mu_{205}, v_{149}, \pi_{143}\right\rangle\left\langle\mu_{232}, v_{192}, \pi_{122}\right\rangle\left\langle\mu_{254}, v_{230}, \pi_{121}\right\rangle
\end{aligned}
$$

After fuzzifying the $R, G, B$ values, matrix (3.7) is obtained.

$$
\begin{gathered}
{\left[R, G, B,\left\{\mu_{r_{i j}}, v_{g_{i j}}, \pi_{b_{i j}}\right\}\right] \equiv} \\
\equiv\left[\begin{array}{ccc}
\langle 0.678,0.356,0.321\rangle & \langle 0.827,0.682,0.466\rangle & \langle 0.968,0.709,0.494\rangle \\
\langle 0.954,0.094,0.258\rangle & \langle 1.000,0.749,0.447\rangle & \langle 0.988,0.886,0.498\rangle \\
\langle 0.854,0.058,0.164\rangle & \langle 0.984,0.721,0.415\rangle & \langle 0.992,0.909,0.462\rangle \\
\langle 0.803,0.584,0.560\rangle & \langle 0.909,0.752,0.478\rangle & \langle 0.996,0.901,0.474\rangle
\end{array}\right]
\end{gathered}
$$

\section{Conclusion and future work}

In this paper, an attempt has been made to represent RGB images using 3 folded - intuitionistic fuzzy index matrixes. Further, the authors planned to define operations on 3 folded - IFIMR using 3D - IFIM operations. Ultimately, these operations will be used to design algorithms for processing RGB images. Also, these algorithms can be developed for processing HSL images.

\section{References}

[1] Traneva, V. (2014). On 3-dimensional intuitionistic fuzzy index matrices. $10^{\text {th }}$ Int. Workshop on IFSs, Banska Bystrica, (29 Sept. 2014). Notes on Intuitionistic Fuzzy Sets, 20(4), 59-64. 
[2] Atanassov, K. (2014). Index Matrices: Towards an Augmented matrix Calculus. Springer International Publishing Switzerland. Studies in Computational Intelligence 573. pp. 30, 95-98.

[3] Atanassov, K. (1994). Index matrix representation of the intuitionistic fuzzy graphs. Proceeding of Fifth Scientific Session of the Math. Foundations of Artificial Intelligence Seminar, Sofia, 36-41. Accessed 5 Oct 1994 (Preprint MRL-MFAIS-10-94).

[4] Atanassov, K. (1987). Generalized index matrices. Comptes rendus de l'Academie Bulgare des Sciences, 40(11), 15-18.

[5] Atanassov, K. (2010). On index matrices, Part 1: Standard cases. Advanced Studies in Contemporary Mathematics, 20(2), 291-302.

[6] Atanassov, K. (2010). On index matrices, Part 2: Intuitionistic fuzzy case. Proceedings of the Jangjeon Mathematical Society, 13(2), 121-126.

[7] Parvathi, R., Thilagavathi, S., Thamizhendhi, G., \& Karunambigai, M. G. (2014). Index matrix representation of intuitionistic fuzzy graphs. Notes Intuitionistic Fuzzy Sets, 20(2), $100-108$. 